\title{
Detecting Target Identity-Location/Response Binding in Visuo-spatial Tasks Using a Cueing Procedure: An Exploratory Study
}

\author{
Ben Kajaste*, Eric Buckolz \\ The University of Western Ontario, London, Canada \\ Email: *bkajaste@uwo.ca
}

How to cite this paper: Kajaste, B., \& Buckolz, E. (2018). Detecting Target Identity-Location/Response Binding in Visuo-spatial Tasks Using a Cueing Procedure: An Exploratory Study. Psychology, 9, 691-704.

https://doi.org/10.4236/psych.2018.94043

Received: March 8, 2018

Accepted: April 17, 2018

Published: April 20, 2018

Copyright (c) 2018 by authors and Scientific Research Publishing Inc. This work is licensed under the Creative Commons Attribution International License (CC BY 4.0).

http://creativecommons.org/licenses/by/4.0/

(c) (i) Open Access

\begin{abstract}
We aimed to determine the suitability of a "new" procedure for detecting the existence of binding between a target's identity and its location/response during its processing on a prime trial in a visuo-spatial task. Importantly, the method involved cueing the impending likelihood of a binding violation on a subsequent probe trial. If the latency increasing impact of a binding violation can be reduced or removed when the cue proved to be valid, RT reductions for Conditions that involved a binding violation would show a larger latency decrease than those which did not (relative to uninformative cue trials). This result pattern did not occur, even though data showed that the cue information was used for preparation (RT [valid cue] $<\mathrm{RT}$ [uninformative cue] $>\mathrm{RT}$ [invalid cue]). Either a target identity-location/response binding does not occur, or advance knowledge of its impending violation does not modulate the violation's latency increase impact. In any event, the use of the cue procedure employed here to detect binding is not a viable one.
\end{abstract}

\section{Keywords}

Target Identity_Location/Response Binding, Cueing

\section{Introduction}

Evidence that the retrieval of earlier stored processing (prime trial) can influence current processing (probe trial) (i.e., sequential effects) has been available for some time in a variety of forms, including the fact that previously incorrect predictions decrease the RT benefit of a current correct prediction (e.g., Geller, 1974), that latency facilitation occurs for repeated target identities and/or their responses (e.g., Keele, 1969), and the existence of a number of inhibitory 
after-effect phenomena (i.e., such as negative priming, and the negative compatibility and inhibition of return effects: e.g., Tipper, 2001; Schlaghecken, Fowley, Sembi, Simmons, \& Whitcomb, 2007). The examination of sequential effects is worthwhile because, depending on the task context in which they are observed, they can reveal the presence of various processing characteristics or properties. For example, there is a sequential processing impact observed when there is a change in the status of an object's identity and/or response when going from being "irrelevant" on the prime trial to being "relevant" on the probe trial. The RT for this status change is significantly longer than when the same relevant processing does not undergo a status change. Notably, this holds whether the prime distractor is visible (non-masked) or phenomenally invisible (masked) (e.g., Fitzgeorge, Buckolz, \& Khan, 2011; Schlaghecken et al., 2007). Seemingly, distractor events are processed automatically, in spite of intentions/instructions not to do so. This processing is then stored, and when later retrieved with the presentation of the probe trial, can interfere with related processing (i.e., the negative priming effect; Neill, Terry, \& Valdes, 1994; Tipper, 2001).

There is yet another sequential effect which, along with the processing characteristic that it reveals (i.e., binding; Hommel, 2007), is of specific interest in this study. In this case, partial repetitions of the prime trial S-R processing on the probe trial in a visual identity task (i.e., repeat stimulus identity, change the response or repeat the response, change stimulus identity) are accompanied by RT elevations relative to when the S-R prime processing is entirely repeated, or entirely changed on the probe. Hommel (e.g., 2004; 2007), along with colleagues (e.g., Hommel, Memelink, Zmigrod, \& Colzato, 2014), have interpreted such findings to indicate that executed (prime) responses bind to their sponsoring target's identity dimensions (e.g., shape, size, colour etc.) and/or to their non-identity features (e.g., location, related response), both when they are relevant or irrelevant to response selection. It follows too from the RT results that overcoming binding violations on the probe trial (i.e., partial repetition probes) comes at a time cost; hence, the shorter latencies for the non-violation conditions (complete change, complete repeat of the prime S-R processing).

Recently, Kajaste and Buckolz (2017) extended the earlier binding results produced with visual identity tasks to visual location-based tasks, where the relevant feature of the designated target object determining response selection is its spatial position. Specifically, Kajaste and Buckolz demonstrated that during the course of prime trial processing, the selected response binds to the relevant location occupied by the prime target object and, less consistently, to the irrelevant target's identity. Actually, a target's identity is indirectly relevant in location tasks in that it is needed to identify the pertinent location for response selection; hence, a target's identity needs to be fully processed. This thorough processing enhances the likelihood that the prime target's identity binds with some aspect of prime target processing in location tasks, and so we pursued this possibility here. Specifically, we examined the possibility that the prime target's identity 
binds to its location, and/or to its location and response as a collective.

To accomplish this goal, we used a procedure that generated four Conditions, formed by which prime trial features were repeated/changed on the probe trial: [1] repeat neither target identity, location, nor response; [2] change target identity, repeat target location and response; [3] repeat target identity, change target location and response; [4] repeat target identity, location, and response. Conditions [1] and [4] did not involve potential binding violations while Conditions [2] and [3] did. Typically, binding is held to be revealed when the RTs for Conditions deemed to include binding violations (e.g., [2] [3]) exceed the latencies produced by Conditions where binding violations have been avoided (e.g., [1] [4]). However, this approach to binding detection is not ideal (Kajaste \& Buckolz, 2017). The point of concern can be illustrated by considering the comparisons between Conditions [2] and [3] with Condition [4]. With Conditions [2] and [3], the slowing effect of actual binding violations may be offset or fully countered by repeating the prime target's identity or its location and response, for Conditions [2] and [3], respectively. As a result of opposing latency effects of a prime feature repeat and a binding violation, the outcome could be that the latencies for Conditions [2] and [3], where binding violation slowing actually occurred, are shorter than for Condition [1]. This would give rise to the faulty conclusion that binding had not occurred during prime trial processing. In the same way, one could argue that slower RTs for the partial feature repeats in Conditions [2] and [3] relative to RTs for Condition [4], arose because of the complete prime trial feature replication in Condition [4] and not because of binding violations in Conditions [2] and [3].

We reasoned that one way to avoid the aforementioned interpretation confounding involved with RT comparisons among Conditions [1] [2] [3] [4] and so allow latency differences to reflect binding existence of the kind studied here would be to introduce both uninformative and informative cues between prime and probe trial pairs. Latencies following an uninformative cue (a "question mark"), typical of past work, would establish baseline RT differences among the four Conditions that would be reflective of their processing demand differences, including whether a binding violation was involved or not. An informative cue (i.e., the numbers 1 - 4) would signal the likely target location (with 75\% accuracy), and so the response needed, on a forthcoming probe trial. Also of note is the fact that whether the prime target identity was repeated or changed on a paired probe trial was fixed (i.e., 100\%). Consequently, while the informative cue did not relate to predicting probe trial target identity, it did forecast when a binding violation would occur on an impending probe trial (Conditions [2] [3]).

With respect to binding detection (target identity to its location/response), the rationale was that if binding did in fact occur for Conditions [2] and [3] and if cueing its impending occurrence on the upcoming probe trial could reduce/eliminate processing delays associated with binding violations, we would see a reduction in RT differences between binding violation and non-binding violation Conditions 
for the validly cued Conditions (i.e., [1] vs. [3] and [2] vs. [4], relative to the uninformative RT differences for these same Condition contrasts. In other words, the RT impact of a valid cue, relative to an uninformative cue, should interact with Conditions, being greater when binding violations had occurred. Naturally, the reason for the absence of such an interaction would be unclear; reflecting either that prime binding had not occurred or, that it had, but is resistant to cue modulation. Note that paired Conditions selected for contrast were chosen on the basis of the fact that the cue forecast a location/response repeat ( [2] [4]) or a location/response change ( [1]).

Actually, there is another aspect to the possibility that validly cueing an impending binding violation could reduce or eliminate the latency impact of unexpected violations that is worthwhile highlighting. It has to do with the recognition that the consequences of prime trial binding to future processing in which they participate are mixed; being "beneficial" in reducing RT size when prime and probe processing demands fully match, but "detrimental" when earlier binding is violated (i.e., partial prime repetition). This maladaptive feature of cognition, which runs counter to the notion that we are evolving into a state of intelligent processing design, has been seen before. For example, consider the phenomenon of negative priming in general (e.g., D'Angelo, Thomson, Tipper, \& Milliken, 2016; Frings, Schneider, \& Fox; 2015) and spatial negative priming (SNP) in particular (e.g., Fitzgeorge, Buckolz, \& Khan, 2011). An SNP effect is observed when the RT for a current (probe) target is significantly greater when it arises at a location formerly occupied by a to-be-ignored distractor, relative to when it occurs at a spatial position that was previously (prime) empty. So, when an irrelevant location (i.e., distractor-occupied) becomes relevant (i.e., target-occupied), target RT is interfered with. Interestingly, the negative influence reflected in the SNP phenomenon can be prevented in a number of ways, including cueing the use of the prime distractor response on the probe trial (e.g., Buckolz, Boulougouris, \& Khan, 2002; Buckolz, Edgar, Kajaste, Lok, \& Khan, 2012; Fitzgeorge \& Buckolz, 2008). It will be interesting to determine whether this same interference prevention is possible with prime trial binding.

To be clear, then, while an important objective here was to look for binding between the prime target's identity and its location/response, of equal importance was to determine whether the cue method to be employed here was suitable for detecting such binding.

Finally, for the sake of convenience, we modified and utilized existing programs to control our procedure. With these programs, Probe Trial Content (either a target + distractor or a target alone) and Probe Trial Content Probabilities (the relative frequencies of these two probe types being .75/.25 or .25/.75 [between-subjects]) were two factors that were manipulated, which generated four distinct "contextual environments" within each we could examine the relationship between Conditions ([1][2][3][4] and Cue Type (valid, uninformative, [invalid]). We had no a priori basis for predicting an impact for these contextual 
environments, other than to note that they can influence spatial negative priming results (e.g., Fitzgeorge et al., 2008).

\section{Methods}

\subsection{Participants \& Apparatus}

Forty-two undergraduate students (22 males, 20 females) participated in this experiment. Participants ages ranged from 19 - 23 years and all reported normal or corrected to normal vision.

Participants were seated at a desk in a dimly lit room $200 \mathrm{~cm}$ from a $61 \mathrm{~cm}(24$ inch) computer monitor which contained the visual display for the experiment. On each trial, a white fixation cross $(.9 \mathrm{~cm}$ in width and height, $.1 \mathrm{~cm}$ thickness) appeared against a black background flanked by two white horizontal bar markers on each side which had the same dimensions as the horizontal component of the fixation cross. The bar markers (denoted L1 - L4 from left to right) were separated from each other and the fixation cross by a distance $.5 \mathrm{~cm}$. This produced a horizontal display distance of $6.5 \mathrm{~cm}$ which resulted in a visual angle of 1.9 degrees.

In order to respond to the appearance of a target stimulus, participants sat with their forearms resting comfortably on the desk with their hands resting on a standard computer keyboard and their middle and index fingers of each hand resting on the keys " $D$ ", "V", "M" and "L". Each of these keys corresponded spatially to a location bar marker on the screen in the visual display (from left to right, respectively). Responses were achieved via finger flexion which depressed the appropriate key and terminated the response interval.

\subsection{Procedure (Data Collection)}

Participants undertook a modified visual spatial negative priming (SNP) task where trials were presented in pairs; first the "prime", and then the "probe". The prime trial always contained a target (green rectangle) and a distractor (red rectangle) object (both measuring $.9 \mathrm{~cm}$ wide $\times 2 \mathrm{~cm}$ high), while the probe trial could contain a target and a distractor (distractor present) or a target by itself (distractor absent). The prime distractor's identity remained unaltered throughout, while that for the prime target could either be repeated (green rectangle) or changed (yellow cross) on the probe. Participants $(n=42)$ were randomly assigned to one of four experimental groups. With group $1(n=12)$, the experimental condition was characterized by the $.75 / .25$ probe distractor probability ratio (.75 distractor present $/ .25$ distractor absent) along with a matching (relative to the prime target's identity) probe target (identity repeat). In group 2 $(n=11)$ a $.25 / .75$ probe distractor probability ratio was utilized (.25 present $/ .75$ absent) along with a matching probe target (identity repeat). For group 3 ( $n=$ $10)$, the experimental manipulation was a $.75 / .25$ probe distractor probability along with a mismatching (relative to the prime target's identity) probe target (identity change). Group $4(n=9)$ undertook a $.25 / .75$ probe distractor proba- 
bility manipulation along with a mismatching probe target (identity change). Participants in the $.75 / .25$ conditions (groups 1 \& 3) completed 2688 randomized trial pairs over 6 sessions with each session lasting approximately $35 \mathrm{mi}-$ nutes. Participants in the $.25 / .75$ conditions (groups $2 \& 4$ ) completed 2880 randomized trial pairs over 6 sessions, each lasting approximately 35 minutes. Participants completed a maximum of one session per day, with no more than one day in between any two sessions. The discrepancy in the number of trials was due to the different trial frequencies associated with the $.75 / .25$ and $.25 / .75$ probe distractor probability manipulations. A complete breakdown of trial type and frequencies for both probe distractor probability conditions can be found in the Appendix.

A trial sequence commenced with a blank screen and an audible warning tone which lasted for $100 \mathrm{~ms}$. This was followed by the appearance of the display paradigm (described above and illustrated in Figure 1) which remained on the screen for the duration of the trial pair. $200 \mathrm{~ms}$ after the appearance of the paradigm, the prime trial objects (target and distractor) appeared at any two of the four possible locations and remained on the screen for $157 \mathrm{~ms}$. A correct response on the prime trial resulted in the paradigm remaining empty for $100 \mathrm{~ms}$, after which the cue (number 1, 2, 3, 4 or "?") appeared in place of the fixation cross and remained on the screen for $400 \mathrm{~ms}$. This cue was informative in that it predicted the location of the upcoming probe target with $75 \%$ accuracy (i.e., cue "1" $=75 \%$ chance that the probe target appears at the first location from left [L1]), except in the case of the "?" cue, which meant the probe target could appear at any one of the four possible locations with all locations being equiprobable. Following the offset of the cue there was a $700 \mathrm{~ms}$ intra-trial-interval during which the paradigm remained empty. Next, the probe trial objects (target-only, or target-plus-distractor) appeared for $157 \mathrm{~ms}$. At the offset of the probe trial the empty paradigm remained on the screen until a response was made or $1000 \mathrm{~ms} \mathrm{had}$ elapsed, at which point the screen went black and a $1500 \mathrm{~ms}$ inter-trial-interval commenced, whose offset was followed by the next warning tone and the beginning of the next trial pair sequence.

Trial pairs that contained errors (button press, anticipations [RT $<100 \mathrm{~ms}$ ] or insufficient vigilance $[\mathrm{RT}>1000 \mathrm{~ms}]$ ) were recorded but were not used in reaction time analyses. Participants were automatically offered a rest break after completion of every 100 trial pairs. Trials resumed when participants pressed the space bar at their discretion.

Before beginning the experimental phase, participants were instructed that 1) they were to respond to any target event with the appropriate key press as quickly and as accurately as possible while ignoring any distractor event should one be present, 2) they were to avoid button press errors and responding before target arrival (i.e., anticipations), 3) trials would be presented in pairs with a cue appearing in between each prime-probe pair, 4) a distractor would appear on the probe trial $75 \%$ of the time or $25 \%$ of the time (depending on which Group they 
were in), and, 5) the cue represented, with $75 \%$ accuracy, the location that the upcoming probe target would appear at, except in the case of the "?" cue, which

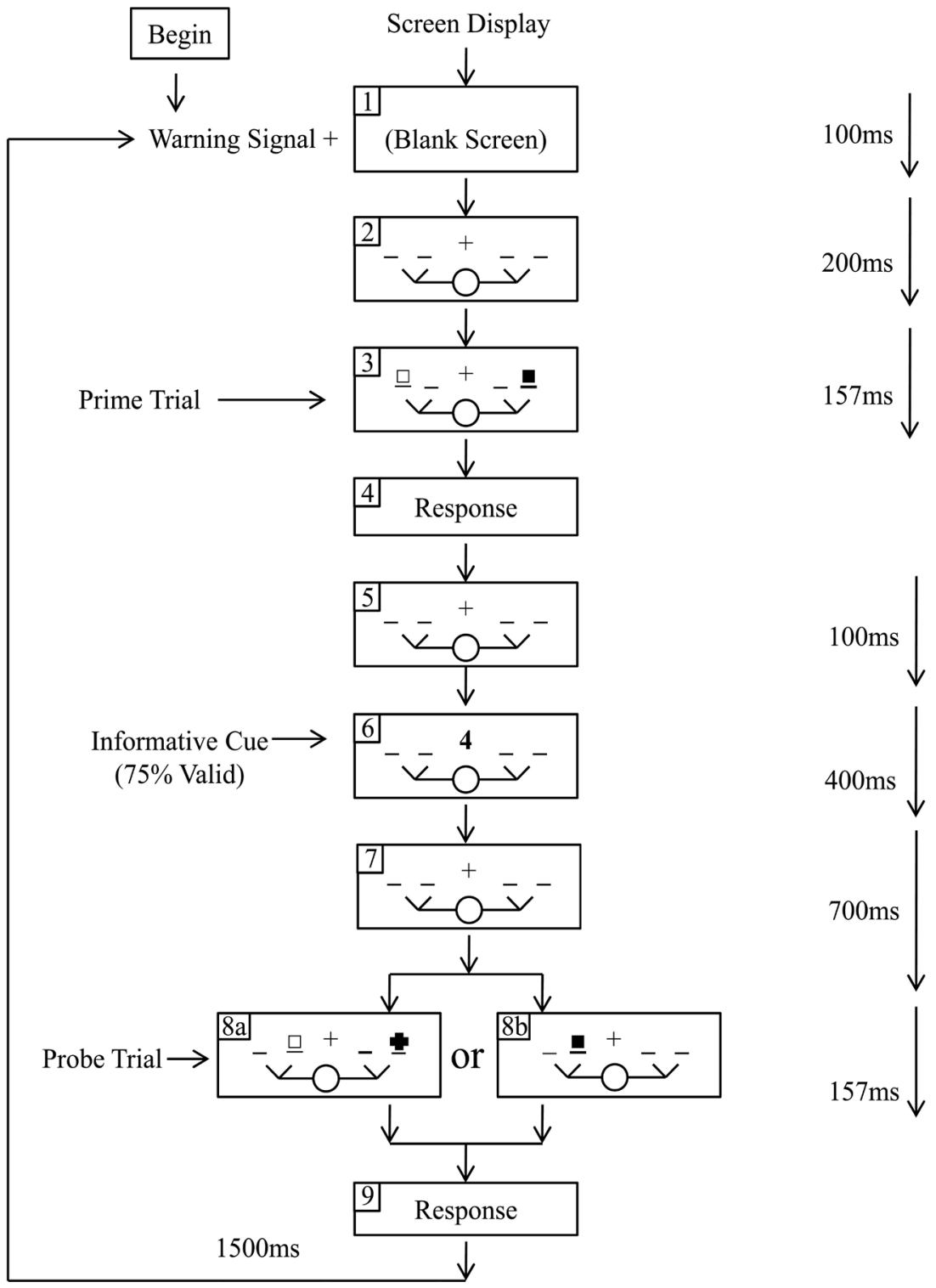

Figure 1. An illustration of a prime-probe trial sequence and presentation durations for the Experiment. Target events could be either a green rectangle (dark rectangle in figure) or a yellow cross (dark cross in figure); only the former could appear on the prime trial, while either target could arise on the probe trial depending on Condition. When a distractor appeared $(100 \%$ on the prime, $75 \%$ or $25 \%$ on the probe respectively, depending on Probe Distractor Probability Condition), it was always a red rectangle (light rectangle in Figure). Panel 6 represents a 75\% valid cue that replaced the fixation cross for $400 \mathrm{~ms}$ between the prime and probe. The number(s) displayed in the cue corresponded spatially to the location bar markers from left to right (i.e. cue "1" = L1 [first location from left]) and represented, with $75 \%$ validity, the likelihood that the probe target would appear at that location. Panel 8a represents a target-plus-distractor probe $(T+D)$ that was validly cued, with target location repeated and target identity changed [2]. Panel $8 \mathrm{~b}$ represents a target-only probe (T-only) that was invalidly cued, with target location changed and target identity repeated [3]. 
meant that the probe target could appear at any of the 4 locations (all equally likely).

Before undertaking the critical portion of the experiment participants completed approximately 10 - 15 prime-probe practice trial pairs and had the opportunity to ask any questions to ensure their understanding of the task requirements.

\section{Results and Discussion}

Individual mean reaction times were utilized for all analyses of variance (ANOVA). Condition Type ([1] vs. [3] or [2] vs. [4]) and Cue Type (valid cue or uninformative cue) served as the main factors, and two ANOVAs were calculated for each of our four contextual environments (.75/.25, $\mathrm{T}+\mathrm{D} ; .75 / .25$, T-only; .25/.75, $\mathrm{T}+\mathrm{D} ; .25 / .75$, T-only). The cell means for these computations are found in Table 1 and Table 2. Recall that the paired Conditions chosen for comparison in each ANOVA were selected on the basis that one contained a binding violation potential, the other did not; and that both have the same cue

Table 1. Mean reaction times (ms) of critical conditions for the $.75 / .25$ probe distractor probability ratio (.75 probe distractor present $/ .25$ probe distractor absent) as a function of probe type (target plus distractor and target-only), and cue type (uninformative, valid [75\%] and invalid [25\%]).

\begin{tabular}{|c|c|c|c|c|c|c|}
\hline \multicolumn{7}{|c|}{$.75 / .25$ Probe Distractor Probability Ratio } \\
\hline \multirow{2}{*}{$\begin{array}{l}\text { Probe } \\
\text { Cue }\end{array}$} & \multicolumn{3}{|c|}{ Target plus Distractor } & \multicolumn{3}{|c|}{ Target-only } \\
\hline & Valid & Uninformative & Invalid & Valid & Uninformative & Invalid \\
\hline \multicolumn{7}{|c|}{ Condition } \\
\hline \multirow{3}{*}[1]{} & 430 & 481 & 512 & 400 & 454 & 499 \\
\hline & (18.5) & (12.8) & $(12.6)$ & $(18.9)$ & $(14.3)$ & $(13.5)$ \\
\hline & $\{4.8\}$ & $\{5.6\}$ & $\{11.0\}$ & $\{5.7\}$ & $\{14.0\}$ & $\{11.3\}$ \\
\hline \multirow{3}{*}{ [3] } & 397 & 455 & 493 & 380 & 442 & 481 \\
\hline & $(17.6)$ & $(11.3)$ & $(10.3)$ & $(16.4)$ & $(9.4)$ & $(11.3)$ \\
\hline & $\{6.0\}$ & $\{10.2\}$ & $\{13.7\}$ & $\{6.8\}$ & $\{16.0\}$ & $\{12.8\}$ \\
\hline $\begin{array}{l}\text { Effect } \\
{[1]-[3]}\end{array}$ & 33 & 26 & 19 & 20 & 12 & 18 \\
\hline \multirow{3}{*}{ [2] } & 434 & 475 & 515 & 403 & 455 & 491 \\
\hline & $(13.8)$ & $(11.2)$ & $(11.3)$ & $(16.0)$ & $(14.2)$ & $(11.6)$ \\
\hline & $\{4.9\}$ & $\{6.9\}$ & $\{9.4\}$ & $\{4.8\}$ & $\{5.0\}$ & $\{8.9\}$ \\
\hline \multirow{3}{*}{ [4] } & 402 & 447 & 483 & 383 & 431 & 465 \\
\hline & $(13.2)$ & (11.9) & $(11.3)$ & $(13.0)$ & (10.5) & $(10.1)$ \\
\hline & $\{5.5\}$ & $\{7.1\}$ & $\{15.3\}$ & $\{6.6\}$ & $\{7.6\}$ & $\{11.3\}$ \\
\hline $\begin{array}{l}\text { Effect } \\
{[2]-[4]}\end{array}$ & 32 & 28 & 32 & 20 & 24 & 26 \\
\hline
\end{tabular}

Notes. ( ) = standard error $(\mathrm{ms}) ;\{\}=$ button press error $\% .{ }^{\star} p<.05$. Conditions (all relative to prime target): $[1]=$ change probe target identity, location and response, $[2]=$ change probe target identity, repeat location and response, [3] = repeat probe target identity, change location and response, [4] = repeat probe target identity, location and response. 
Table 2. Mean reaction times (ms) of critical conditions for the $.25 / .75$ probe distractor probability ratio (.25 probe distractor present $/ .75$ probe distractor absent) as a function of probe type (target plus distractor and target-only), and cue type (uninformative, valid [75\%] and invalid [25\%]).

\begin{tabular}{|c|c|c|c|c|c|c|}
\hline \multicolumn{7}{|c|}{$.25 / .75$ Probe Distractor Probability Ratio } \\
\hline \multirow{2}{*}{$\begin{array}{l}\text { Probe } \\
\text { Cue }\end{array}$} & \multicolumn{3}{|c|}{ Target plus Distractor } & \multicolumn{3}{|c|}{ Target-only } \\
\hline & Valid & Uninformative & Invalid & Valid & Uninformative & Invalid \\
\hline \multicolumn{7}{|c|}{ Condition } \\
\hline \multirow{3}{*}[1]{} & 447 & 480 & 517 & 404 & 452 & 482 \\
\hline & $(14.6)$ & $(14.0)$ & $(16.4)$ & $(12.5)$ & $(11.6)$ & $(13.1)$ \\
\hline & $\{7.9\}$ & $\{8.3\}$ & $\{13.2\}$ & $\{5.9\}$ & $\{14.9\}$ & $\{13.4\}$ \\
\hline \multirow{3}{*}{ [3] } & 426 & 459 & 505 & 392 & 448 & 482 \\
\hline & $(23.8)$ & $(14.4)$ & $(17.0)$ & $(24.0)$ & $(15.0$ & $(11.9)$ \\
\hline & $\{4.5\}$ & $\{9.5\}$ & $\{12.9\}$ & $\{6.0\}$ & $\{16.2\}$ & $\{14.4\}$ \\
\hline $\begin{array}{l}\text { Effect } \\
{[1]-[3]}\end{array}$ & 21 & 21 & 12 & 12 & 4 & 0 \\
\hline \multirow{3}{*}{ [2] } & 450 & 489 & 509 & 401 & 443 & 464 \\
\hline & $(12.1)$ & $(14.8)$ & $(14.3)$ & (9.7) & (10.4) & $(12.9)$ \\
\hline & $\{6.0\}$ & $\{5.1\}$ & $\{12.5\}$ & $\{4.7\}$ & $\{6.2\}$ & $\{9.3\}$ \\
\hline \multirow{3}{*}{ [4] } & 413 & 460 & 490 & 377 & 432 & 454 \\
\hline & $(19.4)$ & $(16.1)$ & $(16.7)$ & $(18.3)$ & $(16.5)$ & $(11.0)$ \\
\hline & $\{3.4\}$ & $\{8.3\}$ & $\{13.3\}$ & $\{4.8\}$ & $\{6.6\}$ & $\{15.9\}$ \\
\hline $\begin{array}{l}\text { Effect } \\
{[2]-[4]}\end{array}$ & 37 & 29 & 19 & 24 & 11 & 10 \\
\hline
\end{tabular}

Notes. ()$=$ standard error $(\mathrm{ms}) ;\{\}=$ button press error $\%{ }^{*} p<.05$. Condition (all relative to prime target): [1] = change probe target identity, location and response, [2] = change probe target identity, repeat location and response, $[3]=$ repeat probe target identity, change location and response, $[4]=$ repeat probe target identity, location and response.

information, that is, whether a repeat or change in the prime target location and response was forecast. Further, the two Conditions used in an ANOVA differed with respect to whether the prime target identity was repeated or changed, a factor that would have to be considered for the interpretation of Condition RT differences. Because target identity repeat or change was entirely predictable, it was uncertain as to what impact this target identity manipulation would have on RT.

In any event, the reasoning was that the RT differences between Conditions following an uninformative cue would establish a baseline value that would reflect all of the processing differences between the Conditions, whatever these may be. When an informative cue turned out to be valid, its only differential impact on the two Conditions involved would be to predict a forthcoming binding violation. If a binding violation time cost had been present with the uninformative cue trials, and if the time cost of a binding violation can be reduced or eliminated when it is validly cued, we should see a greater RT impact of this cue on the binding violation Condition, resulting in a reduced latency difference 
between Conditions in the valid cue instance, relative to when an uninformative cue had been used (i.e., a Condition Type x Cue Type interaction).

The pattern of significance was the same for all eight ANOVAs; throughout, the Cue Type main effect produced significant F-ratios (ranging from 11.59 to 84.22, $p$-values all $<.009$ ), while no significant F-ratios were produced by the Condition Type main effect (ranging from .06 to 2.76, $p$-values ranging from .13 to .81), nor its interaction with Cue Type (all F-ratios were 1 or less). Apparently the contextual environments included in this study do not differentially influence target identity-location/response binding.

These ANOVA outcomes clearly demonstrated that individuals used the informative cue information as probe RTs were significantly faster following valid than uninformative cues (409 msec. vs. $457 \mathrm{msec}$., respectively [Table 1 \& Table 2]). This latency benefit was not selective to Condition Type; however, revealed by the failed Cue Type by Condition Type interactions. Collectively, it follows that the cue information provided was used to prepare the likely required probe response, and/or to process the probe target at a particular spatial position, but not to influence any latency impact of binding violations. Otherwise, valid cue RT benefits should have been larger for Conditions with binding violations than for those without. This was not the case. So, either "target identity-location/response" binding does not occur during prime trial processing, or it does, but its latency elevating impact (upon binding violation) cannot be reduced or removed when the individual knows that an impending probe binding violation is likely.

If we provisionally interpret our findings as indicating that prime target identity does not bind with its location and/or its response in a location task, it would be consistent with results from spatial negative priming tasks which have shown that RT is delayed for ignored-repetition trials (i.e., SNP effect), whether the probe target's identity matches that of the prime distractor or not (e.g., Milliken, Tipper, Houghton, \& Lupianez, 2000). So, whether a prime distractor-location/response binding violation occurs (identity mismatch) or not (identity match), a significant slowing for ignored-repetition trials is observed. The only difference in this study is that it seems that prime target identities do not bind to their location and/or responses either. Actually, a failure of prime target or distractor identities to bind to their locations or responses is less surprising given that these identities randomly appear at all locations during a trial series. Perhaps this circumstance acts to prevent identity bindings?

As it turns out, the non-significant Condition Type main effects here are somewhat puzzling; partly because the numerical RT differences between paired Conditions are quite large (Table 1), and partly because an examination of Newman-Keuls tests applied to the Trial Type by Condition Type interactions revealed significant RT differences between compared Conditions. The latency significance pattern revealed by the Newman-Keuls tests was such that RTs were significantly faster when the prime target identity was repeated, as opposed to when it was changed, on the probe trial. This held despite the fact that target 
identity repetitions or changes were entirely predictable, and despite the fact that identity repetition occurred in a Condition with a binding violation in one instance ( [3]). Seemingly, we cannot cue away the RT benefit of repeating a target's identity on sequential trials, and, the latency facilitation owing to a repetition of the target's prime identity seems to override any RT delays owing to a concurrent binding violation, should one exist.

Another series of ANOVAs were executed, identical to those above, with the only difference being that the invalid cue reaction time data was now included in the Cue Type factor. These eight ANOVAs yielded the same pattern of significance (or lack there of). The Cue Type main effect was significant in all analyses, with F-ratios ranging from 31.79 to 68.40 ( $p$-values all $<.001$ ). The Condition Type main effect (F-ratios ranged from .01 to 2.90, $p$-values all $>.12$ ) as well as the Condition Type $\mathrm{x}$ Cue Type interaction (F-ratios ranging from .03 to 1.03 and $p$-values all $>.38$ ) both failed to achieve significance.

\section{Summary}

We set out to determine whether prime target identities in visuo-spatial tasks bind to their locations and responses, using a new procedure that relied on the latency effects of uninformative and informative cues between Conditions that differed principally with respect to whether they involved a binding violation or not. Paired Conditions selected for comparison were chosen on this basis. Reaction time differences among Conditions following uninformative cues reflected processing duration discrepancies brought on by any disparate processing requirements. An informative cue forecast the likely target location and response on a forthcoming probe trial, as well as predicting the upcoming binding violation in the Conditions where a violation was possible. If a binding violation existed and contributed to RT following uninformative cues, and if a valid cue reduced or eliminated this latency influence, RT differences between binding violation and non-violation Conditions should be less for valid cue than for uninformative trials.

This did not occur. The RT differences between Conditions were unaltered by a valid cue. Either a target's identity does not bind to its location and response or, it does, but the slowing impact of a binding violation cannot be offset when its occurrence is expected. In any event, the cue procedure employed here is unsuitable for detecting target identity-location/response binding existence in visuo-spatial tasks.

On a lesser note, we did replicate a classic cue effect whereby RT (valid cue) < RT (uninformative cue) < RT (invalid cue), reflective of a benefit-cost impact of informative cueing and indicating that our subjects used the cue information provided (e.g., Geller, 1974; 1975). Additionally, we did see that delays encountered when target identity changes occur on successive prime-probe trials could not be removed when they were fully predictable; their related RTs were still significantly slower than for predictable target identity repeats. 


\section{References}

Buckolz, E., Boulougouris, A., \& Khan, M. (2002). Influence of Probe-Trial Selection on the Location Negative Priming Effect. Canadian Journal of Experimental Psychology/Revuecanadienne de psychologie expérimentale, 56, 273.

https://doi.org/10.1037/h0087403

Buckolz, E., Edgar, C., Kajaste, B., Lok, M., \& Khan, M. (2012). Inhibited Prime-Trial Distractor Responses Solely Produce the Visual Spatial Negative Priming Effect. Attention, Perception, \& Psychophysics, 74, 1632-1643.

https://doi.org/10.3758/s13414-012-0366-0

D’Angelo, M. C., Thomson, D. R., Tipper, S. P., \& Milliken, B. (2016). Negative Priming 1985to 2015: A Measure of Inhibition, the Emergence of Alternative Accounts, and the Multipleprocess Challenge. The Quarterly Journal of Experimental Psychology, 69, 1890-1909. https://doi.org/10.1080/17470218.2016.1173077

Fitzgeorge, L., \& Buckolz, E. (2008). Spatial Negative Priming Modulation: The Influence of Probe-Trial Target Cueing, Distractor Presence, and an Intervening Response. European Journal of Cognitive Psychology, 20, 994-1026.

https://doi.org/10.1080/09541440701686250

Fitzgeorge, L., Buckolz, E., \& Khan, M. (2011). Recently Inhibited Responses Are Avoided for both Masked and Nonmasked Primes in a Spatial Negative Priming Task. Attention, Perception, \& Psychophysics, 73, 1435. https://doi.org/10.3758/s13414-011-0125-7

Frings, C., Schneider, K. K., \& Fox, E. (2015). The Negative Priming Paradigm: An Update and Implications for Selective Attention. Psychonomic Bulletin \& Review, 22, 1577-1597. https://doi.org/10.3758/s13423-015-0841-4

Geller, E. S. (1974). Preceding Prediction Outcome and Prediction Outcome Probability: Interacting Determinants of Choice Reaction Time. Journal of Experimental Psychology, 103, 426. https://doi.org/10.1037/h0037154

Geller, E. S. (1975). Prediction Outcome and Choice Reaction Time: Inhibition versus Facilitation Effects. Acta Psychologica, 39, 69-82. https://doi.org/10.1016/0001-6918(75)90022-0

Hommel, B. (2004). Event Files: Feature Binding in and across Perception and Action. Trends Incognitive Sciences, 8, 494-500. https://doi.org/10.1016/j.tics.2004.08.007

Hommel, B. (2007). Feature Integration across Perception and Action: Event Files Affect Response Choice. Psychological Research, 71, 42-63. https://doi.org/10.1007/s00426-005-0035-1

Hommel, B., Memelink, J., Zmigrod, S., \& Colzato, L. S. (2014). Attentional Control of the Creation and Retrieval of Stimulus-Response Bindings. Psychological Research, 78, 520-538. https://doi.org/10.1007/s00426-013-0503-y

Kajaste \& Buckolz (2017). Forced and Freely Selected Responses Bind with Target Object Location in a Visuo-spatial Task (under Review).

Keele, S. W. (1969). Repetition Effect: A Memory-Dependent Process. Journal of Experimental Psychology, 80, 243. https://doi.org/10.1037/h0021173

Milliken, B., Tipper, S. P., Houghton, G., \& Lupiáñez, J. (2000). Attending, Ignoring, and Repetition: On the Relation between Negative Priming and Inhibition of Return. Attention, Perception, \& Psychophysics, 62, 1280-1296.

https://doi.org/10.3758/BF03212130

Neill, W. T., Terry, K. M., \& Valdes, L. A. (1994). Negative Priming without Probe Selection. Psychonomic Bulletin \& Review, 1, 119-121. https://doi.org/10.3758/BF03200767 
Schlaghecken, F., Rowley, L., Sembi, S., Simmons, R., \& Whitcomb, D. (2007). The Negative Compatibility Effect: A Case for Self-Inhibition. Advances in Cognitive Psycholo$g y, 3,227$. https://doi.org/10.2478/v10053-008-0027-y

Tipper, S. P. (2001). Does Negative Priming Reflect Inhibitory Mechanisms? A Review and Integration of Conflicting Views. The Quarterly Journal of Experimental Psychology: Section A, 54, 321-343. https://doi.org/10.1080/713755969 


\section{Appendix}

Trial type frequency breakdown.

\begin{tabular}{|c|c|c|c|c|c|}
\hline \multirow{2}{*}{ Cue } & \multirow{2}{*}{ Probe } & \multicolumn{2}{|c|}{ Condition } & \multicolumn{2}{|c|}{ PDP Ratio } \\
\hline & & PTI Repeat & PTI Change & $.75 / .25$ & $.25 / .75$ \\
\hline Uninformative & $\mathrm{T}+\mathrm{D}$ & Non-Critical & Non-Critical & 192 & 96 \\
\hline Uninformative & $\mathrm{T}+\mathrm{D}$ & {$[4]$} & [2] & 48 & 24 \\
\hline Uninformative & $\mathrm{T}+\mathrm{D}$ & [3] & {$[1]$} & 48 & 24 \\
\hline Uninformative & T-only & Non-Critical & Non-Critical & 24 & 108 \\
\hline Uninformative & T-only & {$[4]$} & {$[2]$} & 24 & 108 \\
\hline Uninformative & T-only & {$[3]$} & {$[1]$} & 48 & 216 \\
\hline Valid (75\%) & $\mathrm{T}+\mathrm{D}$ & Non-Critical & Non-Critical & 864 & 96 \\
\hline Valid (75\%) & $\mathrm{T}+\mathrm{D}$ & {$[4]$} & [2] & 216 & 24 \\
\hline Valid (75\%) & $\mathrm{T}+\mathrm{D}$ & [3] & {$[1]$} & 216 & 24 \\
\hline Valid (75\%) & T-only & Non-Critical & Non-Critical & 108 & 396 \\
\hline Valid (75\%) & T-only & {$[4]$} & [2] & 108 & 396 \\
\hline Valid (75\%) & T-only & {$[3]$} & {$[1]$} & 216 & 792 \\
\hline Invalid (25\%) & $\mathrm{T}+\mathrm{D}$ & Non-Critical & Non-Critical & 336 & 336 \\
\hline Invalid (25\%) & $\mathrm{T}+\mathrm{D}$ & {$[4]$} & [2] & 48 & 48 \\
\hline Invalid (25\%) & $\mathrm{T}+\mathrm{D}$ & {$[3]$} & {$[1]$} & 48 & 48 \\
\hline Invalid (25\%) & T-only & Non-Critical & Non-Critical & 36 & 36 \\
\hline Invalid (25\%) & T-only & {$[4]$} & [2] & 36 & 36 \\
\hline Invalid (25\%) & T-only & {$[3]$} & {$[1]$} & 72 & 72 \\
\hline & & & & Total $=2688$ & Total $=2880$ \\
\hline
\end{tabular}

Notes. PDP Ratio = Probe Distractor Probability Ratio (.75[Probe Distractor Present]/.25[Probe Distractor Absent] or.25[Probe Distractor Present]/.75[Probe Distractor Absent]); T + D = Target-plus-distractor; T-only = Target-only; PTI = Probe Target Identity; Condition (all relative to prime target): [1] = change probe target identity, location and response, $[2]=$ change probe target identity, repeat location and response, $[3]=$ repeat probe target identity, change location and response, $[4]=$ repeat probe target identity, location and response. The Probe Target Identity (repeat or change) was a blocked/between subjects factor, so the total trials for PTI Repeat Conditions would be $2688(.75 / .25)$ and $2880(.25 / .75)$; the total trials for the PTI Change Conditions would also be $2688(.75 / .25)$ and $2880(.25 / .75)$ respectively. 